\title{
PSYCHE CPMG-HSQMBC: An NMR Spectroscopic Method for Precise and Simple Measurement of Long-Range Heteronuclear Coupling Constants
}

\author{
István Timári, ${ }^{[a]}$ László Szilágyi, ${ }^{[b]}$ and Katalin E. Kövér* ${ }^{[a]}$
}

\begin{abstract}
Title change ok? $\square$ Among the NMR spectroscopic parameters, long-range heteronuclear coupling constants convey invaluable information on torsion angles relevant to glycosidic linkages of carbohydrates. A broadband homonuclear-decoupled PSYCHE CPMG-HSQMBC method for the precise and direct measurement of multiple-bond heteronuclear couplings is presented. The PSYCHE scheme built into the pulse sequence efficiently eliminates unwanted proton-proton splittings from the heteronuclear multiplets so that the desired heteronuclear couplings can be determined simply by measuring frequency differences between peak maxima of pure antiphase doublets. Moreover, PSYCHE CPMG-HSQMBC can provide significant improvement in sensitivity as compared to an earlier Zangger-Sterk-based method. Applications of the proposed pulse sequence are demonstrated for the extraction of ${ }^{n} J\left({ }^{1} \mathrm{H}_{,}^{77} \mathrm{Se}\right)$ and ${ }^{n} J\left({ }^{1} \mathrm{H}_{,}^{13} \mathrm{C}\right)$ values, respectively, in carbohydrates; further extensions can be envisioned in any $J$-based structural and conformational studies.
\end{abstract}

Over the years, high-resolution NMR spectroscopy has proved to be the single most important experimental technique to investigate carbohydrate conformations and dynamic properties in solution. ${ }^{[1]}$ The shapes of carbohydrate molecules, specifically those of glycosides and oligosaccharides, are controlled by several intra- and intermolecular interactions; among these the conformation around the glycosidic linkage is of primary importance. Taking, as an example, a disaccharide with regular $\mathrm{O}$ glycosidic linkage, this conformation is described by two torsion angles, $\Phi\left(\mathrm{H} 1-\mathrm{C} 1-\mathrm{O} 1-\mathrm{C}^{\prime} n\right)$ and $\Psi\left(\mathrm{C} 1-\mathrm{O} 1-\mathrm{C}^{\prime} n-\mathrm{H}^{\prime} n\right)$. Further to this most common two-bond glycosidic linkage (C-O-C), two monosaccharides can be connected across three bonds (C-O-

[a] I. Timári, Prof. Dr. K. E. Kövér

Department of Inorganic and Analytical Chemistry

University of Debrecen

Egyetem tér 1, H-4032 Debrecen (Hungary)

E-mail:kover@science.unideb.hu

[b] Prof.Dr. L. Szilágyi

Department of Organic Chemistry

University of Debrecen

Egyetem tér 1, H-4032 Debrecen (Hungary)

Supporting information for this article is available on the WWW under http://dx.doi.org/10.1002/chem.201502641.
C-C) as well. In such cases, three torsion angles, $\Phi, \Psi$, and $\Omega$ are required to describe the conformation around the glycosidic linkage. Further diversity is achieved when the bridging $\mathrm{O}-$ atom in two-bond linkages are replaced by other atoms such as $\mathrm{C}, \mathrm{N}, \mathrm{S}, \mathrm{Se}$, or when two non-O-atoms comprise a threebond glycosidic bridge. ${ }^{[2]}$ Conformational preferences around the $\mathrm{C}-\mathrm{O}$ bonds of pendant $\mathrm{OH}$ groups also contribute to shape the three-dimensional structures and enable intra/intermolecular hydrogen bonding of carbohydrates. These features, which can be disclosed by NMR studies, are of fundamental importance in physiologically relevant intermolecular interactions, such as binding to proteins or hydrolytic cleavage by enzymes or in designing carbohydrate-based pharmaceuticals. ${ }^{[3]}$ Among the NMR parameters, homo- and heteronuclear coupling constants convey valuable information on torsion angles relevant to glycosidic linkages ${ }^{[1 b, 4]}$ or to the distribution of hydroxy and hydroxymethyl rotamers. ${ }^{[5]}$

Determination of heteronuclear multiple-bond couplings remains, however, a challenging task owing to the low sensitivity of the relevant correlation experiments, and also to the fact that long-range heteronuclear $\left({ }^{n} J\left({ }^{1} \mathrm{H}, \mathrm{X}\right)\right)$ and proton-proton coupling constants $\left(J\left({ }^{1} \mathrm{H}^{1}{ }^{1} \mathrm{H}\right)\right)$ are typically the same order of magnitude. Of the several methods reported, ${ }^{[6]}$ the HSQMBC (heteronuclear single quantum multiple-bond correlation) experiment ${ }^{[7]}$ and its variants ${ }^{[8]}$ have found widespread application owing to their general usefulness, good sensitivity, and easy implementation. In the classical HSQMBC-type methods however, the co-evolution of the proton-proton and the multiple-bond proton-heteronucleus coupling interactions during the coupling evolution as well as the acquisition periods may result in severe distortions in the resulting HSQMBC multiplets. Therefore, extraction of the desired heteronuclear coupling constants often requires complex multiplet fitting procedures, or at worst, measurements of the required couplings may be impeded by partial signal cancellations in mixed-phase signals. To minimize this shortcoming of the HSQMBC method, ${ }^{[7]}$ several modifications have been proposed, for example, including CPMG pulse trains, ${ }^{[0]}$ selective $180^{\circ}$ proton pulses, ${ }^{[10]}$ or a perfect echo element ${ }^{[11]}$ in the long-range coupling-matched INEPT block. Recently, the pure in-phase (PIP) module ${ }^{[12]}$ has been introduced in the refocused HSQMBC experiment to avoid undesired phase modulations of multiplets. Nevertheless, these modifications still allow evolution of proton-proton couplings during acquisition, which leads to unwanted protonproton splittings superimposed on the target HSQMBC doublets. 
To overcome the shortcomings mentioned, a band-selective homodecoupled HSQMBC experiment ${ }^{[13]}$ has been proposed. The inherent drawback of this approach is, however, that homonuclear decoupling can be achieved only in regions with non-mutually $J$-coupled proton sites (such as amide regions in peptides). We have recently reported a Zangger-Sterk (ZS)based $^{[14]}$ broadband proton-proton decoupled CPMGHSQMBC method ${ }^{[15]}$ for simple and precise measurement of long-range heteronuclear coupling constants, which is efficient even in molecules with complex proton-proton coupling networks. A common drawback of all ZS-type experiments is however the significant loss of sensitivity owing to slice-selective excitation. Using multiple-frequency shaped pulses ${ }^{[16]}$ or changing the offset of the selective pulse after each scan ${ }^{[17]}$ the sensitivity can be improved, in principle, but to take advantage of these improvements, the nature of the spin systems involved has to be known in advance. The BIRD (bilinear rotation decoupling $)^{[18]}$ isotope selective module (distinguishing between protons attached to ${ }^{13} \mathrm{C}$ or ${ }^{12} \mathrm{C}$ ), which has successfully been utilized without sensitivity penalty for broadband homonuclear decoupling in heteronuclear one-bond correlation experiments, ${ }^{[19]}$ cannot be applied here, because it does not distinguish between remotely (heteronuclear multiple-bond) coupled protons. PSYCHE (pure shift yielded by chirp excitation), ${ }^{[20]}$ proposed recently for broadband homonuclear decoupling, is a conceptually new approach using a pair of small flip angle, frequency-swept chirp pulses in the presence of a weak magnetic field gradient. This technique can provide nearly an order of magnitude enhancement in sensitivity compared to the earlier ZS-based method. ${ }^{[14 b]}$

Herein we report a novel NMR method that combines the PSYCHE approach ${ }^{[20 a]}$ with the CPMG-HSQMBC sequence ${ }^{[9 b]}$ for precise and simple measurement of long-range heteronuclear couplings with significantly improved sensitivity. To illustrate the potential of the experiment, multiple-bond heteronuclear coupling constants have been determined in carbohydrates where extraction of these values from the conventional proton-coupled HSQMBC multiplets would have demanded iterative computer-assisted fittings.

In the proposed method (Figure 1), CPMG pulse trains suppress the proton-proton coupling evolution during the initial INEPT step of the pulse sequence. Between the CPMG$\mathrm{HSQMBC}$ block and acquisition of the $\operatorname{FID}\left(t_{3}\right)$, a second evolution time $\left(t_{2}\right)$, incremented in steps of $1 / \mathrm{sw} 2$, a hard $180^{\circ}$ proton pulse, and a weak field gradient under a pair of small flip angle, swept-frequency Chirp pulses (PSYCHE element) are applied in succession to quench the effects of proton-proton couplings. The PSYCHE element refocusses active spins (which will be observed) in a stimulated echo, while passive spins (coupling partners) remain unaffected. In combination with a hard $180^{\circ}$ proton pulse, passive proton spins are inverted while the active ones are left unaffected. Consequently, the net effect is the continuous evolution of the proton chemical shifts and the heteronuclear couplings throughout $t_{2}$, while the evolution of unwanted homonuclear couplings is refocused at the midpoint of the data acquisition period. As reported earlier, ${ }^{[14 b, 15,21]}$ free induction decay $\left(\operatorname{FID}\left(t_{3}\right)\right)$ is collected in chunks

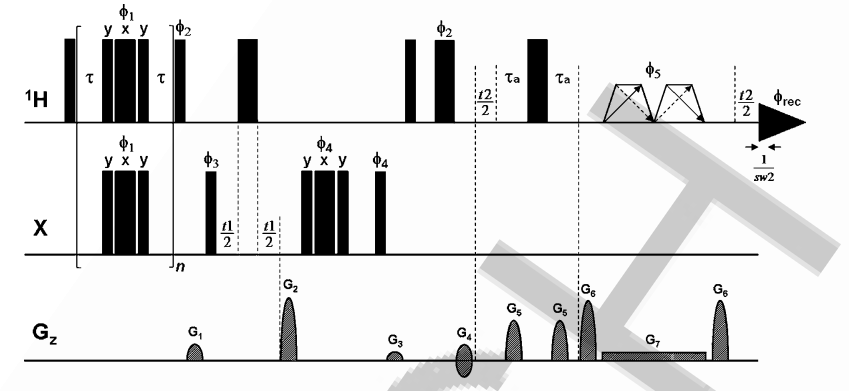

Figure 1. Pulse sequence of the PSYCHE CPMG-HSQMBC experiment proposed for the measurement of long-range heteronuclear coupling constants. Narrow and wide filled bars correspond to $90^{\circ}$ and $180^{\circ}$ pulses respectively, with phase $x$ unless indicated otherwise. Low flip angle ( $\beta$ ), frequency-swept Chirp pulses are shown as trapezoids with diagonal arrows. To improve the sensitivity of the experiment, Chirp pulses which sweep frequency in opposite directions can be used simultaneously, as indicated by the dotted arrows. $\phi_{1}$ is incremented according to XY-16 cycles within the CPMG sequence, thus $n$ should ideally be adjusted to a multiple of 16 . Other phases are $\phi_{2}=y ; \phi_{3}=x,-x ; \phi_{4}=x, x,-x,-x ; \phi_{5}=x, x, x, x, y, y, y, y ;$ and $\phi_{\text {rec }}=x$, $-x,-x, x,-x, x, x,-x$. Delays are set as follows: $\tau=140-150 \mu \mathrm{s}, \tau_{\mathrm{a}}=1 /$ (4.sw2). Coherence order selection and echo-antiecho phase sensitive detection in the $x$ dimension are achieved with gradient pulses $\left(G_{2}\right.$ and $\left.G_{4}\right)$ in the ratio $80: 20.1$ for ${ }^{13} \mathrm{C}$ and $80: 15.257$ for ${ }^{77} \mathrm{Se}$, respectively. Purging gradient pulses $\left(\mathrm{G}_{1}\right.$ and $\left.\mathrm{G}_{3}\right)$ are set to $19 \%, 11 \%$ of maximum gradient strength $\left(50 \mathrm{G} \mathrm{cm}^{-1}\right)$. Coherence selection gradient pulses $\left(\mathrm{G}_{5}\right.$ and $\left.\mathrm{G}_{6}\right)$ used in the extra PSYCHE dimension have $49 \%$ and $77 \%$. Sine-bell-shaped gradient pulses of $1 \mathrm{~ms}$ duration are utilized, followed by a recovery delay of $200 \mu \mathrm{s}$ $\left(G_{1}-G_{4}\right)$ and $1000 \mu s\left(G_{5}, G_{6}\right)$. Weak magnetic field gradient $\left(G_{7}\right)$ used under the Chirp pulses is adjusted for $1.8 \%$ of maximum gradient strength.

(1/sw2) of data, with a duration (typically $10-25 \mathrm{~ms}$ ) that is short on the timescale of $J$ evolution. Prior to regular 2D Fourier transformation, a pseudo 2D dataset (interferogram) with negligible homonuclear $J$ modulation is constructed by concatenating all the data chunks recorded.

To demonstrate the potential of the proposed PSYCHE CPMG-HSQMBC method, multiple-bond ${ }^{1} \mathrm{H}-{ }^{77}$ Se coupling constants $\left({ }^{n}\left({ }^{1} \mathrm{H}^{77} \mathrm{Se}\right)\right)$ were measured in a diglycosyl selenide (I) (Figure 2). The conventional CPMG-HSQMBC spectrum (Figure $2 \mathrm{c}$ ) clearly shows undesired proton-proton splittings superimposed on the target heteronuclear multiplets, preventing direct and precise measurement of long-range heteronuclear couplings in most cases. In contrast, all ${ }^{n} J\left({ }^{1} \mathrm{H}^{77} \mathrm{Se}\right)$ couplings can be easily measured from the pure antiphase HSQMBC doublets obtained using either PSYCHE-based (Figure $2 \mathrm{a}$ ) or ZSbased (Figure $2 \mathrm{~b}$ ) approach for broadband homonuclear decoupling. It is important to note that simplification of HSQMBC multiplets is achieved at the price of sensitivity loss in both proton-decoupled experiments, however, PSYCHE was much less affected and compared to ZS method performed with optimal setting of decoupling parameters yielded nearly three times better signal to noise ratio in this particular case. To compare sensitivities in detail, ZS and PSYCHE ${ }^{1} \mathrm{H}$ spectra of compound I acquired with varying the experimental parameters (bandwidth of RSNOB pulse, strength of slice selection gradient, and flip angle of Chirp pulses) are given in the Supporting Information (Figures S1-S3).

The heteronuclear multiple-bond couplings measured from the two decoupled spectra (data given above and under the 


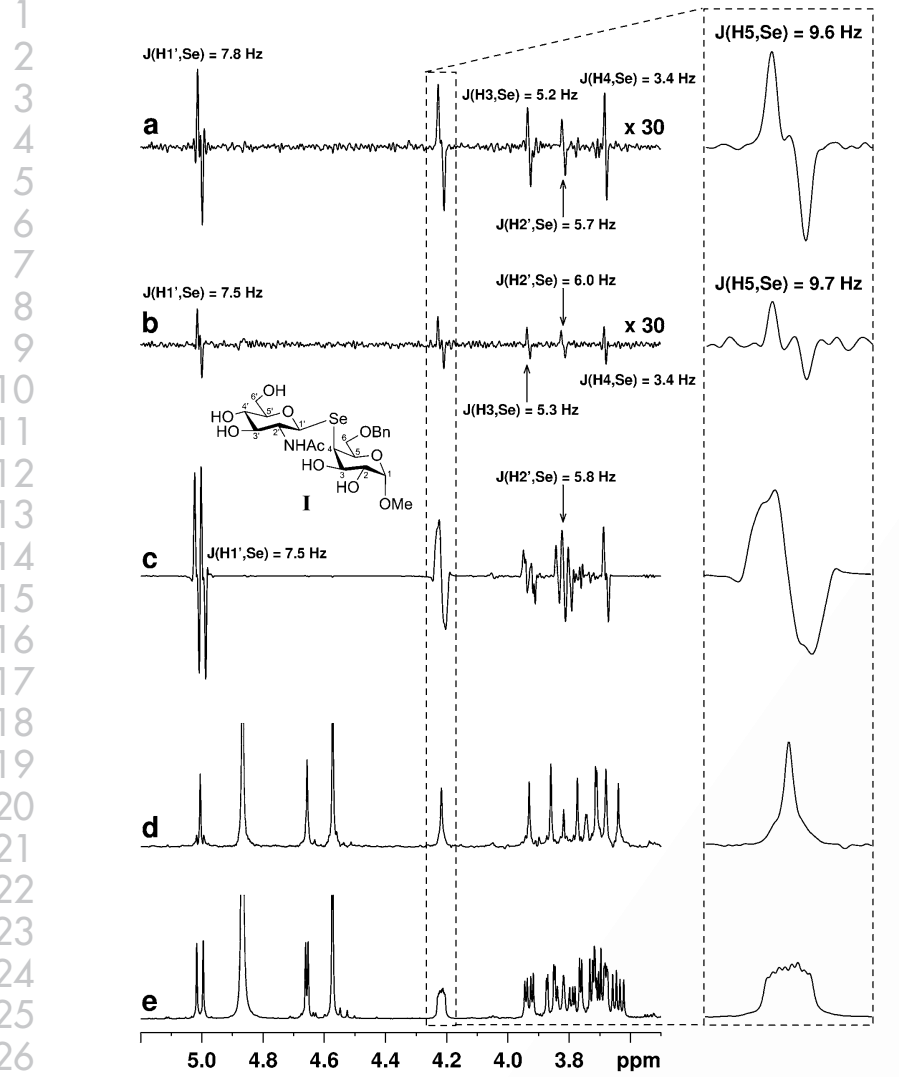

Figure 2. Comparison of ${ }^{1} \mathrm{H}-{ }^{77} \mathrm{Se} C P M G-H S Q M B C$ spectra obtained for a diglycosyl selenide (I), with PSYCHE (a), with ZS (b), and without (c) broadband homonuclear decoupling. Representative PSYCHE ${ }^{1} \mathrm{H}$ (d) and normal ${ }^{1} \mathrm{H}(\mathrm{e})$ NMR spectra of I are also shown. Each of spectra (a), (b), and (c) were recorded with $70.3 \mathrm{~ms}$ of heteronuclear coupling evolution during the initial CPMG-INEPT step and in experiment times of $2 \mathrm{~h} 50 \mathrm{~min}$. Note that the ZS decoupled spectrum (b) was acquired with optimal setting of decoupling parameters (such as bandwidth of the selective pulse and strength of the slice-selection gradient), allowing maximum performance of the experiment.

corresponding multiplets in Figure $2 \mathrm{a}$ and $\mathrm{b}$ ) are in good agreement with each other, and also with those extracted from multiplets obtained using the original CPMG-HSQMBC sequence (Figure $2 \mathrm{C}$ ). This can be taken as a proof that the PSYCHE decoupling element does not affect the magnitudes of heteronuclear couplings. Note, however, that two coupling constants could only be extracted, without using fitting procedures, from the complex multiplets of the conventional CPMG-HSQMBC spectrum.

The less favorable NMR properties of ${ }^{13} \mathrm{C}\left({ }^{77} \mathrm{Se}\right.$ three times more sensitive than ${ }^{13} \mathrm{C}$ ) make the measurement of multiplebond ${ }^{1} \mathrm{H}-{ }^{13} \mathrm{C}$ coupling constants $\left({ }^{n} J\left({ }^{1} \mathrm{H},{ }^{13} \mathrm{C}\right)\right)$ even more challenging at natural ${ }^{13} \mathrm{C}$ abundance. To check the PSYCHE CPMG-HSQMBC method for the determination of ${ }^{n} \mathrm{~J}\left({ }^{1} \mathrm{H},{ }^{13} \mathrm{C}\right)$ as well, a heparin-analogue trisaccharide (II) was selected. The ${ }^{1} \mathrm{H}$ NMR spectrum of II is characterized by closely packed proton multiplets (minimum frequency difference between coupling partners being $<40 \mathrm{~Hz}$; see the Supporting Information, Figure $\mathrm{S} 4$ for complete ${ }^{1} \mathrm{H}$ assignment). For comparison of sensitivities, a ZS-decoupled CPMG-HSQMBC spectrum was also recorded with the same measurement time (Figure 3). In-

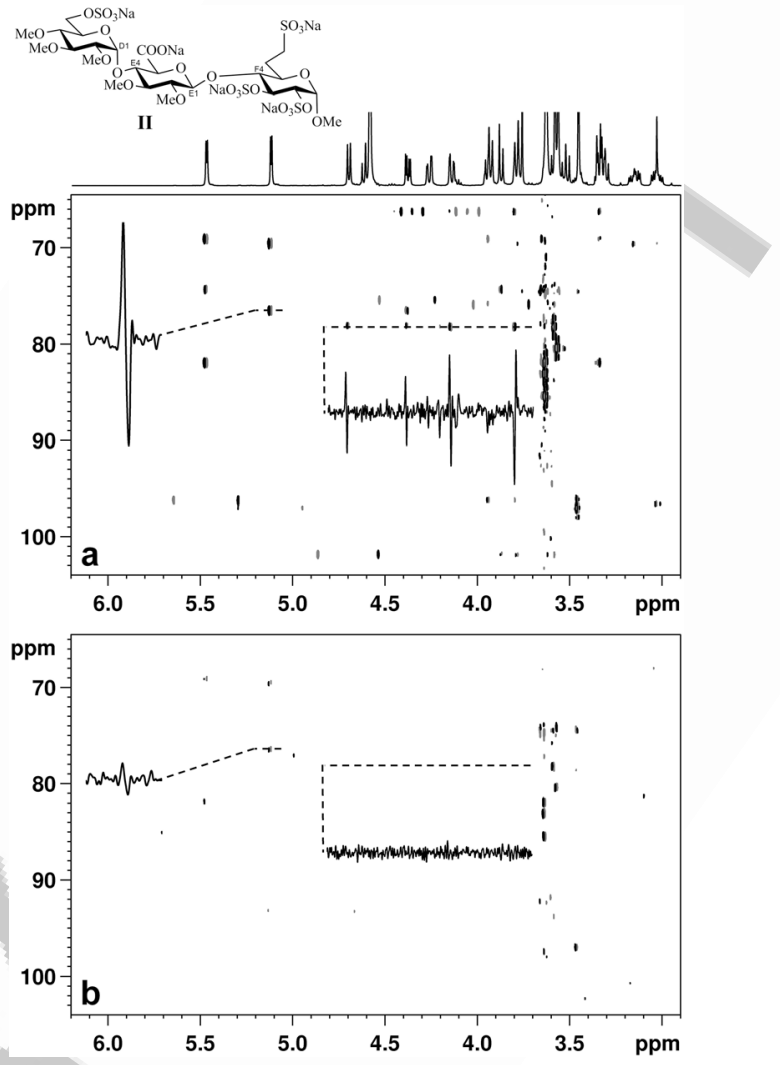

Figure 3. Comparison of contour plots and extracted rows of PSYCHE (a) and ZS (b) ${ }^{1} \mathrm{H}-{ }^{13} \mathrm{C}$ CPMG-HSQMBC spectra obtained for a heparin-analogue trisaccharide (II). Representative ${ }^{1} \mathrm{H}$ NMR spectrum of II above the $2 \mathrm{D}$ contour plots is also shown. Both spectra (a) and (b) were recorded with $83.7 \mathrm{~ms}$ of heteronuclear coupling evolution during the initial CPMG-INEPT step and in experiment times of $32 \mathrm{~h}$. Other experimental details are given in the Supporting Information.

spection of the results clearly shows a lot more correlations in the PSYCHE spectrum (Figure $3 \mathrm{a}$ ) as compared to the ZS-decoupled one (Figure $3 \mathrm{~b}$ ), and signals appearing in both are, on average, seven-fold stronger in the PSYCHE spectrum. The weaker efficiency of the ZS-type experiment can mainly be ascribed to the small frequency difference of protons to be decoupled, necessitating the use of highly selective pulse with narrow bandwidth (of ca. 70 ms duration) to achieve efficient decoupling, which in turn reduces the slice thickness and hence the sensitivity (see the Supporting Information, Figures S4 and S5 for sensitivity comparison of ZS and PSYCHE ${ }^{1} \mathrm{H}$ spectra of compound II.) On the contrary, the nature (characteristics) of the spin systems involved has no effect on the efficiency of the PSYCHE CPMG-HSQMBC experiment. The compromise between sensitivity and spectral purity in the PSYCHE technique is simply controlled by adjusting the flip angle of Chirp pulses, ${ }^{[20 a]}$ which is illustrated by representative PSYCHE ${ }^{1} \mathrm{H}$ spectra of compounds I and II in the Supporting Information, Figures S3 and S6, respectively. Importantly, all four interglycosidic ${ }^{3} J\left({ }^{1} \mathrm{H},{ }^{13} \mathrm{C}\right)$ couplings in the studied trisaccharide could be measured with ease and high precision from the PSYCHE CPMG-HSQMBC spectrum (Supporting Information, Figure S7a), while only one of them was detectable and meas- 
urable from the ZS-decoupled spectrum. Note that precise determination of these couplings is not feasible from the protoncoupled multiplets of the conventional CPMG-HSQMBC experiment (Supporting Information, Figure S7b).

In conclusion, a broadband homonuclear decoupled NMR method has been developed for the direct and precise measurement of long-range heteronuclear coupling constants. PSYCHE approach built-in the CPMG-HSQMBC sequence effectively removes undesired proton-proton splittings from the heteronuclear multiplets to allow measurement of multiplebond heteronuclear couplings with ease from the pure antiphase doublets. Besides, PSYCHE CPMG-HSQMBC can provide significant enhancement in sensitivity compared to the earlier ZS-based method, ${ }^{[15]}$ and can be applied without tedious finetuning from sample to sample. Further improvement in sensitivity can be achieved by using cryoprobes. The efficiency of the experiment proposed has been illustrated on carbohydrates, including measurement of ${ }^{3} \mathrm{~J}\left({ }^{1} \mathrm{H}^{13}{ }^{13} \mathrm{C}\right)$ values. These data are conveying valuable information on the conformations around the glycosidic linkages. Extraction of these couplings from the conventional proton-coupled HSQMBC multiplets would demand computer-aided fitting procedures. Importantly, application of PSYCHE CPMG-HSQMBC is not limited to carbohydrates, it can be put to good advantage for measuring ${ }^{n} J\left({ }^{1} \mathrm{H}, \mathrm{X}\right)$ values in structural studies of any kind of small and medium-sized molecules with extended protonproton coupling networks.

\section{Acknowledgements}

The authors thank Sára Balla for her skilful technical assistance in the preparation of samples. Dr. Anikó Borbás and Dr. Mihály Herczeg are acknowledged for their generous gift of heparinanalogue trisaccharide. Financial support from OTKA K 105459 and OTKA NN 109671 (to K.E.K. and L.Sz.) and from Richter Gedeon Talentum Alapítvány (Ph.D. scholarship to I.T.) is gratefully acknowledged.

\section{Keywords: broadband homonuclear decoupling} carbohydrates - HSQMBC · long-range heteronuclear coupling • NMR spectroscopy

[1] a) J. Ø. Duus, C. H. Gotfredsen, K. Bock, Chem. Rev. 2000, 100, 4589 4614 ; b) K. E. Kövér, L. Szilágyi, G. Batta, D. Uhrín, J. Jiménez-Barbero, in
Comprehensive Natural Products II Chemistry and Biology, Vol. 9 (Eds.: L. Mander, H.-W. Lui), Elsevier, Oxford, 2010, pp. 197-246.

[2] L. Szilágyi, O. Varela, Curr. Org. Chem. 2006, 10, 1745-1770.

[3] D. Solís, N. V. Bovin, A. P. Davis, J. Jiménez-Barbero, A. Romero, R. Roy, K. Smetana Jr, H.-J. Gabius, Biochim. Biophys. Acta, Gen. Subj. 2015, 1850, $186-235$

[4] K. E. Kövér, A. A. Kumar, Y. Y. Rusakov, L. B. Krivdin, T. Z. Illyés, L. Szilágyi, Magn. Reson. Chem. 2011, 49, 190-194.

[5] K. E. Kövér, T. Beke, A. Lipták, A. Perczel, J. Comput. Chem. 2009, 30, $540-550$.

[6] a) B. L. Marquez, W. H. Gerwick, R. T. Williamson, Magn. Reson. Chem 2001, 39, 499-530; b) T. Parella, J. F. Espinosa, Prog. Nucl. Magn. Reson. Spectrosc. 2013, 73, 17-55.

[7] R. T. Williamson, B. L. Marquez, W. H. Gerwick, K. E. Kövér, Magn. Reson Chem. 2000, 38, 265-273.

[8] L. Castañar, T. Parella, in Annual Reports on NMR Spectroscopy, Vol. 84 (Ed.: A. W. Graham), Academic Press, 2015, pp. 163-232.

[9] a) H. Koskela, I. Kilpelainen, S. Heikkinen, J. Magn. Reson. 2003, 164, $228-232$; b) K. E. Kövér, G. Batta, K. Fehér, J. Magn. Reson. 2006, 181, 89-97; c) S. Boros, K. E. Kövér, Magn. Reson. Chem. 2011, 49, 106-110.

[10] a) S. Gil, J. F. Espinosa, T. Parella, J. Magn. Reson. 2011, 213, 145-150; b) J. Saurí, J. F. Espinosa, T. Parella, Angew. Chem. 2012, 124, 3985-3988; Angew. Chem. Int. Ed. 2012, 51, 3919-3922.

[11] B. Baishya, C. L. Khetrapal, J. Magn. Reson. 2014, 242, 143-154.

[12] L. Castañar, J. Saurí, R. T. Williamson, A. Virgili, T. Parella, Angew. Chem. 2014, 126, 8519-8522; Angew. Chem. Int. Ed. 2014, 53, 8379-8382.

[13] L. Castañar, J. Saurí, P. Nolis, A. Virgili, T. Parella, J. Magn. Reson. 2014, $238,63-69$.

[14] a) K. Zangger, H. Sterk, J. Magn. Reson. 1997, 124, 486-489; b) J. A Aguilar, S. Faulkner, M. Nilsson, G. A. Morris, Angew. Chem. 2010, 122, 3993-3995; Angew. Chem. Int. Ed. 2010, 49, 3901 - 3903.

[15] I. Timári, T. Z. Illyés, R. W. Adams, M. Nilsson, L. Szilágyi, G. A. Morris, K. E. Kövér, Chem. Eur. J. 2015, 21, 3472-3479.

[16] L. Castañar, P. Nolis, A. Virgili, T. Parella, Chem. Eur. J. 2013, 19, $15472-$ 15475.

[17] P. Sakhaii, B. Haase, W. Bermel, R. Kerssebaum, G. E. Wagner, K. Zangger, J. Magn. Reson. 2013, 233, $92-95$.

[18] J. R. Garbow, D. P. Weitekamp, A. Pines, Chem. Phys. Lett. 1982, 93, $504-$ 509.

[19] a) L. Paudel, R. W. Adams, P. Király, J. A. Aguilar, M. Foroozandeh, M. J. Cliff, M. Nilsson, P. Sándor, J. P. Waltho, G. A. Morris, Angew. Chem. 2013, 125, 11830-11833; Angew. Chem. Int. Ed. 2013, 52, 11616-11619; b) P. Kiraly, R. W. Adams, L. Paudel, M. Foroozandeh, J. A. Aguilar, I. Timári, M. J. Cliff, M. Nilsson, P. Sándor, G. Batta, J.P. Waltho, K. E. Kövér, G. A. Morris, J. Biomol. NMR 2015, 62, 43-52.

[20] a) M. Foroozandeh, R. W. Adams, N. J. Meharry, D. Jeannerat, M. Nilsson, G. A. Morris, Angew. Chem. 2014, 126, 7110-7112; Angew. Chem. Int. Ed. 2014, 53, 6990-6992; b) M. Foroozandeh, R. W. Adams, M. Nilsson, G. A. Morris, J. Am. Chem. Soc. 2014, 136, 11867-11869.

[21] I. Timári, L. Kaltschnee, A. Kolmer, R. W. Adams, M. Nilsson, C. M. Thiele, G. A. Morris, K. E. Kövér, J. Magn. Reson. 2014, 239, 130-138.

Received: July 6, 2015

Published online on $\square \square$ ul, 0000 


\section{COMMUNICATION}

A PSYCHE sequence incorporated in the CPMG-HSQMBC NMR experiment for broadband homonuclear decoupling efficiently removes undesired protonproton splittings from heteronuclear multiplets. This method allows longrange heteronuclear couplings of interest to be determined simply by measuring frequency differences between maxima of pure antiphase doublets.
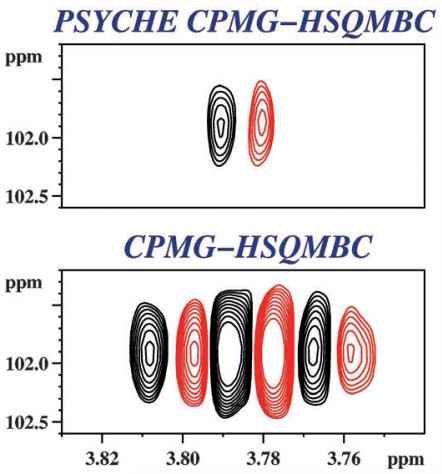

NMR Spectroscopy

I. Timári, L. Szilágyi, K. E. Kövér*

\section{$\mathbf{\square}-\mathbf{\square}$}

PSYCHE CPMG-HSQMBC: An NMR Spectroscopic Method for Precise and Simple Measurement of Long-Range Heteronuclear Coupling Constants 